\title{
CLASSIFICATION OF ORTHOGROUPS AS SEMILATTICES OF VARIOUS RECTANGULAR GROUPS FULFILLING $x^{p+1} \approx x$
}

\author{
ALEXANDER JENDE
}

\begin{abstract}
Completely regular semigroups whose idempotents form a subsemigroup are called orthodox completely regular semigroups, or briefly orthogroups. In this paper, we characterize orthogroups which are semilattices of various rectangular groups fulfilling $x^{p+1} \approx x$ for a fixed prime number $p$ by disjunctions of identities.
\end{abstract}

\section{INTRODUCTION}

A semigroup $S$ is called a completely regular semigroup if $S$ is a union of groups. Due to the work of Clifford ([2]), Fantham ([5]), Petrich ([10-12]) and others, there are a great deal of facts known about this special class of semigroups. Completely regular semigroups whose idempotents form a subsemigroup are called orthodox completely regular semigroups, or briefly orthogroups. For this special class of semigroups, there is a characterization given by Yamada ([16]), which uses a more general result of Clifford ([3]). Yamada showed that an orthogroup is a semilattice of rectangular groups. In particular, any band is a semilattice of rectangular bands as McLean has proven ([8]).

In 2013, R. A. R. Monzo ([9]) characterized a semilattice of rectangular bands and groups with exponent 2 by the so called inclusion classes. This improves the decomposition of orthogroups in a special case (see also [1], [14]).

At this point, it seems to be natural to ask for the characterization of semigroups which are semilattices of certain classes of orthogroups. We will introduce some notation for classes of orthogroups, to be used in this paper:

$L_{0}$ : the trivial semigroup,

$L_{1}$ : class of all right zero semigroups,

$L_{2}$ : class of all left zero semigroups,

$L_{3}$ : class of all rectangular bands,

$L_{4}$ : class of all groups with prime exponent $p$,

$L_{5}$ : class of all right groups with prime exponent $p$,

$L_{6}$ : class of all left groups with prime exponent $p$,

$L_{7}$ : class of all rectangular groups with exponent $p$,

where $p \in \mathbb{N}$ is an arbitrary but fixed prime number. Based on the result by Monzo, for a fixed prime number $p$ and a nonempty subset $I \subseteq\{1,2,3,4,5,6\}$, we

MSC (2010): primary 08B15, 20M10, $20 \mathrm{M} 19$.

Keywords: semilattices, orthogroups, disjunctions of identities, rectangular groups, Rees matrix semigroup. 
characterize the class $C$ of all semigroups $S$ such that every $S$ is a semilattice $Y$ of semigroups $S_{\alpha}$ with $S_{\alpha} \in \bigcup_{i \in I} L_{i}$ for $\alpha \in Y$ by disjunctions of identities. More precisely, we give a set $A=\left\{u_{j} \approx v_{j}: j \in J\right\}$ where the equations $u_{j} \approx v_{j}, j \in J$ are generated from the variables $x$ and $y$ such that

$$
C=\operatorname{MOD}\left(A,\left\{x^{p+1} \approx x\right\}\right)
$$

holds.

Thus, we start our investigations in section 2 with some basic definitions and results concerning semilattices of semigroups, completely simple semigroups (in particular Rees matrix semigroups) and disjunctions of identities. In section 3, we give some conditions for sets of equations to describe orthodox semigroups fulfilling $x^{p+1} \approx x$. Furthermore, we investigate some properties of disjunctions of identities and concentrate on their connection to classes of rectangular groups. Finally, we prove the main result by giving an explicit characterization.

\section{Preliminaries}

For the standard terminology and notation of semigroup theory, we refer the reader to [6]. In particular, we denote by $E_{S}$ the set of all idempotent elements for a semigroup $S$.

Let $A$ be a non-empty subset of a semigroup $S$, then $A$ is called left ideal if $s \in S, a \in A$ imply $s a \in A ; A$ is called a right ideal if $s \in S, a \in A$ imply as $\in A$; and $A$ is called an ideal, if $A$ is both a left ideal and a right ideal. If $S$ is the only ideal of the semigroup $S$ and there exists an element $e \in E_{S}$ such that, whenever $e f=f e=f$ for an element $f \in E_{S}$, we have $e=f$, then $S$ is called a completely simple semigroup. For investigations of this latter kind of semigroups, we use the well-known result of Suschkewitsch (1928) based on the Rees Theorem ([13]):

Theorem 2.1. [6] Let $G$ be a group, let $I, \Lambda$ be non-empty sets and let $P=\left(p_{\lambda i}\right)$ be a $\Lambda \times I$ matrix with entries in $G$. Let $S=(I \times G \times \Lambda)$, with a multiplication on $S$ defined by

$$
(i, a, \lambda)(j, b, \mu):=\left(i, a p_{\mu j} b, \mu\right) .
$$

Then, $S$ is a completely simple semigroup.

Conversely, every completely simple semigroup is isomorphic to a semigroup constructed in this way.

These so defined semigroups are called Rees matrix semigroups (over the group $G$ ). Because of our focus on orthodox semigoups, there is a simpler form of the previous theorem:

Theorem 2.2. Let $G$ be a group, let $I, \Lambda$ be non-empty sets.

Let $S=I \times G \times \Lambda$, and define a multiplication on $S$ by

$$
(i, a, \lambda)(j, b, \mu)=(i, a b, \mu) .
$$

Then, $S$ is an orthodox completely simple semigroup.

Conversely, every orthodox completely simple semigroup is isomorphic to a semigroup constructed in this way. 
An orthodox completely simple semigroup is also called a rectangular group. So, every $S \in L_{i}$ for $i \in\{0,1,2,3,4,5,6,7\}$ is isomorphic to an orthodox Rees matrix semigroup. We can easily see that the previous set of semigroup classes is partially ordered, and thus can be illustrated in the following way:

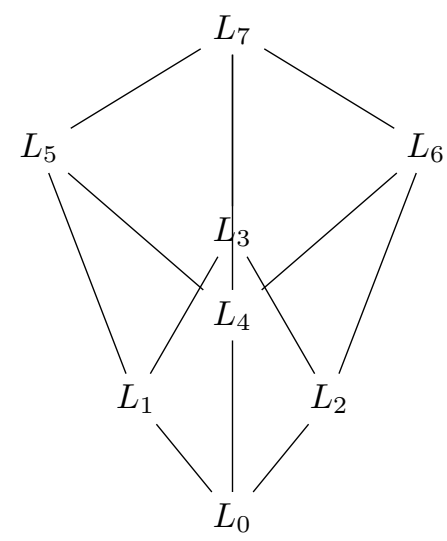

Figure 1. Lattice of elements in $L_{7}$.

Recall that a semigroup $S$ is called a semilattice if $S$ is commutative and every element is idempotent. A semigroup $S$ is a semilattice $Y$ of semigroups $S_{\alpha}$ if there exists a homomorphism $\phi$ of $S$ onto the semilattice $Y$ such that $S_{\alpha}=\phi^{-1}(\alpha)$ for all $\alpha \in Y$. The link between the completely simple semigroups and the completely regular semigroups is a fundamental theorem by Clifford, which is basic for the below results:

Theorem 2.3. [6] Every completely regular semigroup is a semilattice of completely simple semigroups.

For more detailed information on completely regular semigroups and orthogroups, we refer the reader to [6].

Our classification of particular orthogroups as semilattices of special rectangular groups is based on the concept of disjunctions of identities. The following definition of this concept is taken from Thron and Koppitz ([15]) and related to the inclusion classes of Monzo as well as to the definitions by Ljapin ([7]) and Evseev ([4]).

Let $X=\{x, y, z, \ldots\}$ be a non-empty set and let $X^{+}$be the set of all words generated by the elements of $X$. For every semigroup $S$ and every map $h: X \longrightarrow$ $S$, there is a unique extension of $h$ to a homomorphism $\bar{h}: X^{+} \longrightarrow S$.

A set $D \subseteq X^{+} \times X^{+}$is said to be a disjunction of identities in $S$ (in symbols: $S \vDash D)$ if for all maps $h: X \longrightarrow S$ there is $u \approx v \in D$ with $\bar{h}(u)=\bar{h}(v)$ where we use $u \approx v$ instead of $(u, v) \in X^{+} \times X^{+}$. A set $\mathcal{D} \subseteq \mathcal{P}\left(X^{+} \times X^{+}\right)$holds in $S$ (in symbols: $S \in M O D(\mathcal{D}))$ if $S \vDash D$ for all $D \in \mathcal{D}$.

Let $u \in X^{+}$be a term with $u=u_{1} \ldots u_{m}$ for $u_{1}, \ldots, u_{m} \in X$ and $m \in \mathbb{N}$. Then, the leftmost variable of $u$ is defined by leftmost $(u):=u_{1}$. Dually, we have 
the definition of the rightmost variable of $u$ by rightmost $(u):=u_{m}$. Furthermore, the length of $u$ is given by $l(u):=m$. The change of a word $u$ is defined by

$$
r(u):=\left|\left\{i \in\{1, \ldots, m-1\}: u_{i} \neq u_{i+1}\right\}\right| .
$$

We use this latter notion to describe the following properties on $X^{+} \times X^{+}$:

$$
\begin{aligned}
& P_{1}=\left\{u \approx v \in X^{+} \times X^{+}: \text {leftmost }(u)=\text { leftmost }(v)\right\}, \\
& P_{2}=\left\{u \approx v \in X^{+} \times X^{+}: \text {rightmost }(u)=\text { rightmost }(v)\right\}, \\
& P_{3}=\left\{u \approx v \in X^{+} \times X^{+}: l(u) \equiv l(v) \bmod p\right\} \\
& P_{4}=\left\{u \approx v \in X^{+} \times X^{+}: r(u) \equiv r(v) \bmod p\right\} .
\end{aligned}
$$

In the following investigations, we only use a two-element set $X$ with the elements $x$ and $y$, i.e. $X=\{x, y\}$.

\section{Classification of Orthogroups as semilattices of various RECTANGULAR GROUPS FULFILLING $x^{p+1} \approx x$}

Lemma 3.1. Let $u$ be a word built up by the variables $x$ and $y$.

If leftmost $(u) \neq$ rightmost $(u)$, then $r(u)$ is odd.

If leftmost $(u)=$ rightmost $(u)$, then $r(u)$ is even.

Proof. Without loss of generality, assume in the first case leftmost $(u)=x$. This gives us

$$
u=x^{k_{1}} y^{k_{2}} \ldots x^{k_{r(u)}} y^{k_{r(u)+1}}
$$

for $k_{1}, \ldots, k_{r(u)+1} \in \mathbb{N}$, and so $r(u)+1$ is an even number. Hence $r(u)$ is odd. In an analogous way, we prove the second statement.

The following Lemma is based upon some remarks by Howie ([6]):

Lemma 3.2. Let $S$ be a semigroup with $S \vDash\left\{x^{p+1} \approx x\right\}$, then $S$ is completely regular.

Proof. Let $S \vDash\left\{x^{p+1} \approx x\right\}$. Then, for an arbitrary mapping $h:\{x, y\} \longrightarrow S$ with $x \longmapsto a$ for some $a \in S$, there is $\bar{h}(x)=\bar{h}\left(x^{p+1}\right)$. Hence, we observe $a=a^{p+1}$ and so $a=a^{q p+1}$ for $q \geq 0$. For every $s \in \mathbb{N}$, we can write $s=1+q p+u$ where $q \geq 0$ and $0 \leq u \leq p-1$, so it follows $a^{s}=a^{1+q p+u}=a^{1+q p} a^{u}=a^{1+u}$.

For the element $a$, we investigate the set of all powers $K_{a}=\left\{a, a^{2}, a^{3} \ldots\right\}$. Using the previous facts, $K_{a}$ can be limited to $K_{a}=\left\{a, a^{2}, \ldots, a^{p}\right\}$. Let $a^{u+1}, a^{v+1} \in$ $K_{a}$ be arbitrary elements with $0 \leq u, v \leq p-1$, then we can find an element $a^{w+1}$ in $K_{a}$ such that $a^{u+1} a^{w+1}=a^{v+1}$ choosing $w \equiv v-u-1(\bmod p)$ with $0 \leq w \leq p-1$. Obviously, $K_{a}$ is a subgroup of $S$ and so $S$ is an union of groups. Hence, $S$ is a completely regular semigroup.

Lemma 3.3. Let $A:=\left\{u_{j} \approx v_{j}: j \in J\right\}$ be a disjunction of identities where the equations $u_{j} \approx v_{j} \in A, j \in J$, built up by the variables $x$ and $y$. Furthermore, let $u_{j} \approx v_{j} \notin P_{1} \cap P_{2} \cap P_{3}$ for $j \in J$. If there is at most one $j \in J$ with $u_{j} \approx v_{j} \in P_{1} \cap P_{2}$ and $S \in\left(A,\left\{x^{p+1} \approx x\right\}\right)$, then $S$ is orthodox. 
Proof. Let $e, f \in S$ be idempotents. Suppose $u_{j} \approx v_{j} \notin P_{1} \cap P_{2} \cap P_{4}$ for all $j \in J$. Then, we consider the mapping $h:\{x, y\} \longmapsto S$ defined by $h(x)=e$ and $h(y)=f$. So, there is an $j \in J$ such that $\bar{h}\left(u_{j}\right)=\bar{h}\left(v_{j}\right)$.

Admit $u_{j} \approx v_{j} \in P_{1} \cap P_{2}$, then we get $u_{j} \approx v_{j} \notin P_{4}$. We put $u:=x u_{j} y$ and $v:=x v_{j} y$. Hence, without loss of generality, we can assume leftmost $(u)=x$ and rightmost $(u)=y$ such that $\bar{h}(u)=(e f)^{\frac{r(u)+1}{2}}$ and $\bar{h}(v)=(e f)^{\frac{r(v)+1}{2}}$. The last fact works due to Lemma 3.1. Since $u_{j} \approx v_{j} \notin P_{4}$, we conclude $u \approx v \notin P_{4}$. In addition, we have $e f=(e f)^{p+1}$. Thus, $(e f)^{r(u)+1}=(e f)^{r(v)+1}$ by $\bar{h}(u)^{2}=\bar{h}(v)^{2}$ provides $e f=(e f)^{2}$.

Admit $u_{j} \approx v_{j} \notin P_{1}$. Without loss of generality, let $x=\operatorname{leftmost}\left(u_{j}\right) \neq$ leftmost $\left(v_{j}\right)=y$. From $\bar{h}\left(u_{j}\right)=\bar{h}\left(v_{j}\right)$, we obtain $f \bar{h}\left(u_{j}\right)=\bar{h}\left(v_{j}\right)$, i.e. $f \bar{h}\left(u_{j}\right)=$ $\bar{h}\left(u_{j}\right)$ and so $e f \bar{h}\left(u_{j}\right)=\bar{h}\left(u_{j}\right)$. It is easy to see that there is a number $r \in \mathbb{N}$ such that $\bar{h}\left(u_{j}\right) f=(e f)^{r} \bar{h}\left(u_{j}\right) f=(e f)^{p+1}=$ ef. Hence, ef $=\bar{h}\left(u_{j}\right) f=$ $e f \bar{h}\left(u_{j}\right) f=(e f)^{2}$.

Admit $u_{j} \approx v_{j} \notin P_{2}$. Then, we get $e f=(e f)^{2}$ by dual arguments.

Suppose $u_{j} \approx v_{j} \notin\left(P_{1} \cap P_{2}\right) \backslash P_{4}$ for all $j \in J$. Then, we consider the mapping $h:\{x, y\} \longmapsto S$ defined by $h(x)=$ efe and $h(y)=f e f$. So, there is an element $j \in J$ such that $\bar{h}\left(u_{j}\right)=\bar{h}\left(v_{j}\right)$.

Admit $u_{j} \approx v_{j} \in P_{1} \cap P_{2}$, then we get $u_{j} \approx v_{j} \in P_{4}$. As already mentioned above, without loss of generality, we can assume that leftmost $\left(u_{j}\right)=$ $x$ and rightmost $\left(u_{j}\right)=y$ such that $\bar{h}\left(u_{j}\right)=(e f)^{\frac{r\left(u_{j}\right)+1}{2}+l\left(u_{j}\right)}$ and $\bar{h}\left(v_{j}\right)=$ $(e f)^{\frac{r\left(v_{j}\right)+1}{2}+l\left(v_{j}\right)}$ with $u_{j} \approx v_{j} \in P_{4}$. Furthermore, we have ef $=(e f)^{p+1}$, which provides us $(e f)^{l\left(u_{j}\right)}=(e f)^{l\left(v_{j}\right)}$. So, we get $e f=(e f)^{2}$ because $u_{j} \approx v_{j} \notin P_{3}$ and ef $=(e f)^{p+1}$.

Admit $u_{j} \approx v_{j} \notin P_{1} \cap P_{2}$, then we get $e f=(e f)^{2}$ by the same arguments as for the case $h(x)=e$ and $h(y)=f$.

Notice that, since there is $u_{j} \approx v_{j} \in P_{1} \cap P_{2}$ for at most one $j \in J$, all possible cases are investigated. To sum up, it has been shown that $e f \in E_{S}$ for $e, f \in E_{S}$, and so the semigroup $S$ is orthodox.

Lemma 3.4. Let $S$ be an orthodox Rees matrix semigroup over the group $G$ with $G \in L_{4}$. Further, let $h:\{x, y\} \longmapsto S$ and let $u \approx v$ be an equation built up by the variables $x$ and $y$ such that $h(x)=\left(i_{1}, g, \lambda_{1}\right), h(y)=\left(i_{2}, g, \lambda_{2}\right)$ and $\bar{h}(u)=\bar{h}(v)$. Then, the following statements are true:

(1) If $u \approx v \notin P_{1}$, then $i_{1}=i_{2}$,

(2) If $u \approx v \notin P_{2}$, then $\lambda_{1}=\lambda_{2}$,

(3) If $u \approx v \notin P_{3}$, then $g=e$ for the identity $e$ of $G$.

Proof. (1) Without loss of generality, we assume that leftmost $(u)=x$ and thus leftmost $(v)=y$. Hence, we get $\left(i_{1}, g^{l(u)}, \mu_{1}\right)=\bar{h}(u)=\bar{h}(v)=$ $\left(i_{2}, g^{l(v)}, \mu_{2}\right)$ for $\mu_{1}, \mu_{2} \in\left\{\lambda_{1}, \lambda_{2}\right\}$. This implies $i_{1}=i_{2}$.

(2) This is is done dually.

(3) We get by $\bar{h}(u)=\bar{h}(v)$ the equality $\left(j_{1}, g^{l(u)}, \mu_{1}\right)=\left(j_{2}, g^{l(v)}, \mu_{2}\right)$ with $j_{1}, j_{2} \in\left\{i_{1}, i_{2}\right\}$ and $\mu_{1}, \mu_{2} \in\left\{\lambda_{1}, \lambda_{2}\right\}$. Then, $g^{l(u)}=g^{l(v)}$. It is well 
known in group theory that $g=g^{p+1}$ and $g^{l(u)}=g^{l(v)}$ implies that $g$ is the identity in $G$ whenever $p$ is a prime number and $u \approx v \notin P_{3}$.

Definition 3.5. Let

$$
\Phi:\{x, y\}^{+} \times\{x, y\}^{+} \longrightarrow\{0,1,2,3,4,5,6,7\}
$$

be a mapping defined by

$$
\begin{aligned}
& \Phi(u \approx v):=0 \text { if } u \approx v \notin P_{1} \cup P_{2} \cup P_{3}, \\
& \Phi(u \approx v):=1 \text { if } u \approx v \in P_{2} \backslash\left(P_{1} \cup P_{3}\right), \\
& \Phi(u \approx v):=2 \text { if } u \approx v \in P_{1} \backslash\left(P_{2} \cup P_{3}\right), \\
& \Phi(u \approx v):=3 \text { if } u \approx v \in\left(P_{1} \cap P_{2}\right) \backslash P_{3}, \\
& \Phi(u \approx v):=4 \text { if } u \approx v \in P_{3} \backslash\left(P_{1} \cup P_{2}\right), \\
& \Phi(u \approx v):=5 \text { if } u \approx v \in\left(P_{2} \cap P_{3}\right) \backslash P_{1}, \\
& \Phi(u \approx v):=6 \text { if } u \approx v \in\left(P_{1} \cap P_{3}\right) \backslash P_{2}, \\
& \Phi(u \approx v):=7 \text { if } u \approx v \in P_{1} \cap P_{2} \cap P_{3},
\end{aligned}
$$

for all $u, v \in\{x, y\}^{+}$.

It is easy to see that the mapping $\Phi$ is well-defined.

Lemma 3.6. Let $A:=\left\{u_{j} \approx v_{j}: j \in J\right\}$ be a disjunction of identities where the equations $u_{j} \approx v_{j}, j \in J$, built up by the variables $x$ and $y$. If $S$ is a non-trivial rectangular group with

$$
S \in \operatorname{MOD}\left(A,\left\{x^{p+1} \approx x\right\}\right)
$$

then

$$
S \in \bigcup_{j \in J} L_{\Phi\left(u_{j} \approx v_{j}\right)} .
$$

Proof. Since $S \in \operatorname{MOD}\left(\left\{x^{p+1} \approx x\right\}\right)$ is a rectangular group, $S$ is isomorphic to an orthodox Rees matrix semigroup over the group $G$ with $G \in L_{4}$, i.e. $S \in L_{7}$. Assume that $S \notin L_{\Phi\left(u_{j} \approx v_{j}\right)}$ for all $j \in J$. Since $S \cong I \times G \times \Lambda$ is non-trivial, we have $|I|>1$ or $|G|>1$ or $|\Lambda|>1$, which comprises seven cases:

Case 1: $|I|>1,|G|>1$ and $|\Lambda|>1$ : There are $i_{1}, i_{2} \in I$ with $i_{1} \neq i_{2}, \lambda_{1}, \lambda_{2} \in \Lambda$ with $\lambda_{1} \neq \lambda_{2}$ and $g \in G \backslash\{e\}$. Let $h:\{x, y\} \longmapsto I \times G \times \Lambda$ with $h: x \longmapsto\left(i_{1}, g, \lambda_{1}\right)$ and $h: y \longmapsto\left(i_{2}, g, \lambda_{2}\right)$. Since $S \cong I \times G \times \Lambda$ with $S \in \operatorname{MOD}(A)$, there is an element $k \in J$ with $\bar{h}\left(u_{k}\right)=\bar{h}\left(v_{k}\right)$.

Because $S \in L_{7}$, we conclude $\Phi\left(u_{k} \approx v_{k}\right) \neq 7$, i.e. the following condition is fulfilled: $u_{k} \approx v_{k} \notin P_{1} \cap P_{2} \cap P_{3}$. Then, by Lemma 3.4 we get $i_{1}=i_{2}$ or $\lambda_{1}=\lambda_{2}$ or $g=e$. So, there is a contradiction.

Case 2: $|I|=1,|G|>1$ and $|\Lambda|>1$ : There are elements $i_{1} \in I, \lambda_{1}, \lambda_{2} \in \Lambda$ with $\lambda_{1} \neq \lambda_{2}$ and $g \in G \backslash\{e\}$. Let $h:\{x, y\} \longmapsto I \times G \times \Lambda$ with $h: x \longmapsto$ $\left(i_{1}, g, \lambda_{1}\right)$ and $h: y \longmapsto\left(i_{1}, g, \lambda_{2}\right)$. Since $S \cong I \times G \times \Lambda$ with $S \in M O D(A)$, there is an element $k \in J$ with $\bar{h}\left(u_{k}\right)=\bar{h}\left(v_{k}\right)$. From $|I|=1,|G|>1$ and $|\Lambda|>1$, it follows $S \in L_{5}$ and thus $\Phi\left(u_{k} \approx v_{k}\right) \neq 5$. Moreover, 
$S \in L_{5} \subseteq L_{7}$ implies $\Phi\left(u_{k} \approx v_{k}\right) \neq 7$. Thus, we have $u_{k} \approx v_{k} \notin P_{2} \cap P_{3}$. Lemma 3.4 yields $\lambda_{1}=\lambda_{2}$ or $g=e$, once more a contradiction.

Case 3: $|I|>1,|G|>1$ and $|\Lambda|=1$ : Dually, we can verify that this case leads to a contradiction.

Case 4: $|I|>1,|G|=1$ and $|\Lambda|>1$ : There are elements $i_{1}, i_{2} \in I$ with $i_{1} \neq i_{2}$, $e \in G$ and $\lambda_{1}, \lambda_{2} \in \Lambda$ with $\lambda_{1} \neq \lambda_{2}$. Let $h:\{x, y\} \longmapsto I \times G \times \Lambda$ with $h: x \longmapsto\left(i_{1}, e, \lambda_{1}\right)$ and $h: y \longmapsto\left(i_{2}, e, \lambda_{2}\right)$. Since $S \cong I \times G \times \Lambda$ with $S \in \operatorname{MOD}(A)$, there is an element $k \in J$ with $\bar{h}\left(u_{k}\right)=\bar{h}\left(v_{k}\right)$. From $|I|>1,|G|=1$ and $|\Lambda|>1$, it follows $S \in L_{3}$ and thus $\Phi\left(u_{k} \approx v_{k}\right) \neq 3$. Moreover, $S \in L_{3} \subseteq L_{7}$ implies $\Phi\left(u_{k} \approx v_{k}\right) \neq 7$. Thus, we have $u_{k} \approx v_{k} \notin$ $P_{1} \cap P_{2}$. And so we have $i_{1}=i_{2}$ or $\lambda_{1}=\lambda_{2}$ by Lemma 3.4, a contradiction.

Case 5: $|I|=|\Lambda|=1$ and $|G|>1$ : There are elements $i_{1} \in I, \lambda_{1} \in \Lambda$ and $g \in G \backslash\{e\}$. Let $h:\{x, y\} \longmapsto I \times G \times \Lambda$ with $h: x \longmapsto\left(i_{1}, g, \lambda_{1}\right)$ and $h: y \longmapsto\left(i_{1}, g, \lambda_{1}\right)$. Since $S \cong I \times G \times \Lambda$ with $S \in M O D(A)$, there is an element $k \in J$ with $\bar{h}\left(u_{k}\right)=\bar{h}\left(v_{k}\right)$. From $|I|=|\Lambda|=1$ and $|G|>1$, it follows $S \in L_{4}$. Notice that $S \in L_{5}, S \in L_{6}$ and $S \in L_{7}$, which leads to $\Phi\left(u_{k} \approx v_{k}\right) \notin\{4,5,6,7\}$. We conclude $u_{k} \approx v_{k} \notin P_{3}$. By Lemma 3.4, we obtain $g=e$, a contradiction.

Case 6: $|I|=|G|=1$ and $|\Lambda|>1$ : There are elements $i_{1} \in I$ and $\lambda_{1}, \lambda_{2} \in \Lambda$ with $\lambda_{1} \neq \lambda_{2}$. We define a mapping $h:\{x, y\} \longmapsto I \times G \times \Lambda$ with $h: x \longmapsto\left(i_{1}, e, \lambda_{1}\right)$ and $h: y \longmapsto\left(i_{1}, e, \lambda_{2}\right)$. Since $S \cong I \times G \times \Lambda$ with $S \in \operatorname{MOD}(A)$, there is an element $k \in J$ with $\bar{h}\left(u_{k}\right)=\bar{h}\left(v_{k}\right)$. From $|I|=1=|G|$ and $|\Lambda|>1$ it follows $S \in L_{1}$, and thus $S \in L_{3}$ , $S \in L_{5}$ and $S \in L_{7}$. This requires $\Phi\left(u_{k} \approx v_{k}\right) \notin\{1,3,5,7\}$ and we conclude $u_{k} \approx v_{k} \notin P_{2}$. This means $\lambda_{1} \neq \lambda_{2}$ by Lemma 3.4, which is a contradiction.

Case 7: $|\Lambda|=|G|=1$ and $|I|>1$ : Dually, we can verify that this case leads to a contradiction, too.

Altogether, we have shown that $S \notin L_{\Phi\left(u_{j} \approx v_{j}\right)}$ for all $j \in J$ provides a contradiction. Thus, there is an element $j \in J$ with $S \in L_{\Phi\left(u_{j} \approx v_{j}\right)}$, which proves the assertion.

Lemma 3.7. Let $S$ be a semilattice $Y$ of completely simple semigroups $S_{\alpha}$ $(\alpha \in Y)$ such that $S \vDash\left\{x^{p+1} \approx x\right\}$. Further, let $a \in S_{\alpha}$ and $b \in S_{\beta}$ for $\alpha, \beta \in Y$.

(1) If $S_{\alpha \beta} \in L_{1}$, then $a b=b a b$,

(2) If $S_{\alpha \beta} \in L_{2}$, then $a b=a b a$,

(3) If $S_{\alpha \beta} \in L_{3}$, then $a b=a b a^{2 p-2} b$,

(4) If $S_{\alpha \beta} \in L_{5}$, then $a b=b^{p} a b$,

(5) If $S_{\alpha \beta} \in L_{6}$, then $a b=a b a^{p}$,

(6) If $S_{\alpha \beta} \in L_{4}$, then $a b=b^{p} a b a^{p}$,

(7) If $S_{\alpha \beta} \in L_{7}$, then $a b=a b^{p} a^{p} b$.

Proof. (1) Notice that $a b, b a b \in S_{\alpha \beta}$ and so there is $a b=(b a b) a b=$ $b(a b)^{2}=b a b$.

(2) This is done in much the same way.

(3) Notice that $a b, a^{2 p-2} b \in S_{\alpha \beta}$. Thus, we calculate $a b=(a b)^{2}$ $=a b\left(a^{2 p-2} b\right) a b=a b a^{2 p-3}(a b)^{2}=a b a^{2 p-2} b$. 
(4) Notice that $(b a)^{p}$ is an idempotent element in $S_{\alpha \beta} \in L_{5}$ such that $a b=$ $(b a)^{p} a b=b^{p}(b a)^{p} a b=b^{p} a b$.

(5) This is done in much the same way.

(6) We have $a b=a b a^{p}$ because $S_{\alpha \beta} \in L_{4} \subseteq L_{6}$ and $a b=b^{p} a b$ because $S_{\alpha \beta} \in L_{4} \subseteq L_{5}$, respectively. Thus, $a b=a b a^{p}=b^{p} a b a^{p}$.

(7) Notice that $a b,(b a)^{p} \in S_{\alpha \beta}$ with $(b a)^{p}$ idempotent, so $a b=(a b)^{p+1}=$ $a b(a b)^{p-1} a b=a b^{p+1}(a b)^{p-1} a^{p+1} b=a b^{p}(b a)^{p} a^{p} b=a b^{p} a^{p} b$.

In connection with the previous results, we define the following mapping for further purposes:

Definition 3.8. Let

$$
\rho:\{1,2,3,4,5,6\} \longrightarrow\{x, y\}^{+}
$$

be a mapping defined by

$$
\begin{aligned}
& \rho(1):=y x y, \quad \rho(2):=x y x, \rho(3):=x y x^{2 p-2} y, \\
& \rho(4):=y^{p} x y x^{p}, \rho(5):=y^{p} x y, \rho(6):=x y x^{p} .
\end{aligned}
$$

The mapping $\rho$ is well defined.

Lemma 3.9. Let $i \in\{1,2,3,4,5,6\}$, then

$$
L_{\Phi(x y \approx \rho(i))}=L_{i} .
$$

Proof. We consider $\rho(i)$, and observe that $x y \approx \rho(i) \in \Phi^{-1}(i)$ by simple calculations. This implies $\Phi(x y \approx \rho(i)) \in \Phi \Phi^{-1}(i)=\{i\}$ and thus $L_{\Phi(x y \approx \rho(i))}=$ $L_{i}$.

Theorem 3.10. Let $I \subseteq\{1,2,3,4,5,6\}$ be a non-empty set and let $C$ be a class of all semigroups $S$ such that every $S$ is a semilattice $Y$ of semigroups $S_{\alpha}$ with $S_{\alpha} \in \bigcup_{i \in I} L_{i}$ for $\alpha \in Y$. Then, there is a disjunction of identities

$$
A:=\left\{u_{j} \approx v_{j}: j \in J\right\}
$$

such that

$$
C=\operatorname{MOD}\left(A,\left\{x^{p+1} \approx x\right\}\right) .
$$

Proof. Let $I \subseteq\{1,2,3,4,5,6\}$ be a non-empty set, $C$ be the class of all semigroups $S$ such that every $S$ is a semilattice $Y$ of semigroups $S_{\alpha}$ with $S_{\alpha} \in \bigcup_{i \in I} L_{i}$ for $\alpha \in Y$ and let $A$ be defined by $A:=\{x y \approx \rho(i): i \in I\}$.

From this definition of $A$, it follows that $C=\operatorname{MOD}\left(A,\left\{x^{p+1} \approx x\right\}\right)$.

$C \subseteq M O D\left(A,\left\{x^{p+1} \approx x\right\}\right):$ Let $S \in C$. For some $a, b \in S$ we choose a mapping $h:\{x, y\} \longmapsto S$ with $h(x)=a$ and $h(y)=b$. Then, there are $\alpha, \beta \in Y$ such that $a \in S_{\alpha}$ and $b \in S_{\beta}$, and $a b \in S_{\alpha \beta}$. Thus, there is an element $i \in I$ with $S_{\alpha \beta} \in L_{i}$. Then, we conclude that $a b=\bar{h}(\rho(i))$ by Lemma 3.7, which shows that $S \vDash A$. Furthermore, all elements $a \in S$ fulfill $a^{p+1}=a$, hence $S \in \operatorname{MOD}\left(A,\left\{x^{p+1} \approx x\right\}\right)$.

$\operatorname{MOD}\left(A,\left\{x^{p+1} \approx x\right\}\right) \subseteq C$ : Let $S \in \operatorname{MOD}\left(A,\left\{x^{p+1} \approx x\right\}\right)$. Then, $S \vDash$ $\left\{x^{p+1} \approx x\right\}$ and $S$ is a completely regular semigroup by Lemma 3.2. Applying Theorem 2.3 shows that $S$ is a semilattice $Y$ of semigroups $S_{\alpha}$ for $\alpha \in Y$ where 
every $S_{\alpha}$ is isomorphic to a Rees Matrix semigroup (Theorem 2.1). Furthermore, the set $A=\{x y \approx \rho(i): i \in I\}$ fulfills all requirements of Lemma 3.3 and so $S$ is an orthodox semigroup. Particularly, $S$ is a semilattice $Y$ of orthodox semigroups $S_{\alpha}(\alpha \in Y)$ where every $S_{\alpha}$ is a rectangular group as described in Theorem 2.2. This fact makes it possible to use Lemma 3.6, which yields $S_{\alpha} \in \bigcup_{i \in I} L_{\Phi(x y \approx \rho(i))}$ for any $\alpha \in Y$. Hence, by Lemma 3.9 we obtain $S_{\alpha} \in \bigcup_{i \in I} L_{i}$ for all $\alpha \in Y$.

Acknowledgement. The author thanks the referee for the helpful comments and suggestions.

\section{REFERENCES}

[1] J.P. Auraujo and M. Kinyon, A natural characterization of semilattices of rectangular bands and groups of exponent two, Semigroup Forum 91 (2015), 295-298.

[2] A. H. Clifford, The structure of orthodox unions of groups, Semigroup Forum 3 (1972), 283-337.

[3] A. H. Clifford, Semigroups admitting relative inverses, Ann. of Math. (2) 42 (1941), 10371049.

[4] A. E. Evseev, Semigroups with some power identity inclusions, Algebraic Systems with One Relation, Interuniv. Collect. Sci. Works, Leningrad (1985), 21-32.

[5] P. H. H. Fantham, On the classification of a certain type of semigroup, Proc. London Math. Soc., III. Ser. 10 (1960), 409-427.

[6] J. M. Howie, Fundamentals of Semigroup Theory, Oxford University Press, 1995.

[7] E.S. Ljapin, Atoms in the lattice of identical-inclusion varieties of semigroups, Sib. Math. J. 16 (1975), 938-943.

[8] D. McLean, Idempotent semigroups, Amer. Math. Monthly 61 (1954), 110-113.

[9] R. A. R. Monzo, Semilattices of rectangular bands and groups of order two, preprint 2013, arXiv:1301.0828.

[10] M. Petrich, On the varieties of completely regular semigroups, Semigroup Forum 25 (1982), 153-169.

[11] M. Petrich, A structure theorem for completely regular semigroups, Proc. Amer. Math. Soc. 99 (1987), 617-622.

[12] M. Petrich, The structure of completely regular semigroups, Trans. Amer. Math. Soc. 189 (1974), 211-236.

[13] D. Rees, On semi-groups, Proc. Cambridge Philos. Soc. 36 (1940), 387-400.

[14] Y. Shao and M. Ren, On semilattices of rectangular bands and groups, Semigroup Forum 93 (2016), 201-204.

[15] R. Thron and J. Koppitz, Free systems of algebras and ultraclosed classes, Acta Math. Univ. Comenian. (New. Ser.) $\mathbf{7 5}$ (2006), 127-136.

[16] M. Yamada, Strictly inversive semigroups, Bull. Shimane Univ. Natur. Sci. 13 (1963), 128138.

Alexander Jende, University of Potsdam, Institute of Mathematics, Campus Golm, Haus 9, Karl-Liebknecht-Straße 24-25, 14476 Potsdam, Germany

e-mail: alexander.jende@gmx.de 
